Physical Sciences | Raffaella Demichelis

\section{Modelling the growth of minerals}

What do precious gems, seashells, and bones have in common? The answer is minerals. Minerals are chemically complex solids that are mostly formed naturally by Earth's and space's geological processes but can also be produced by many living species, including humans. Dr Raffaella Demichelis, at Curtin University, Australia, is using computationa chemistry to see how these minerals form and how their structure and composito affect their properties and chemical behaviour.
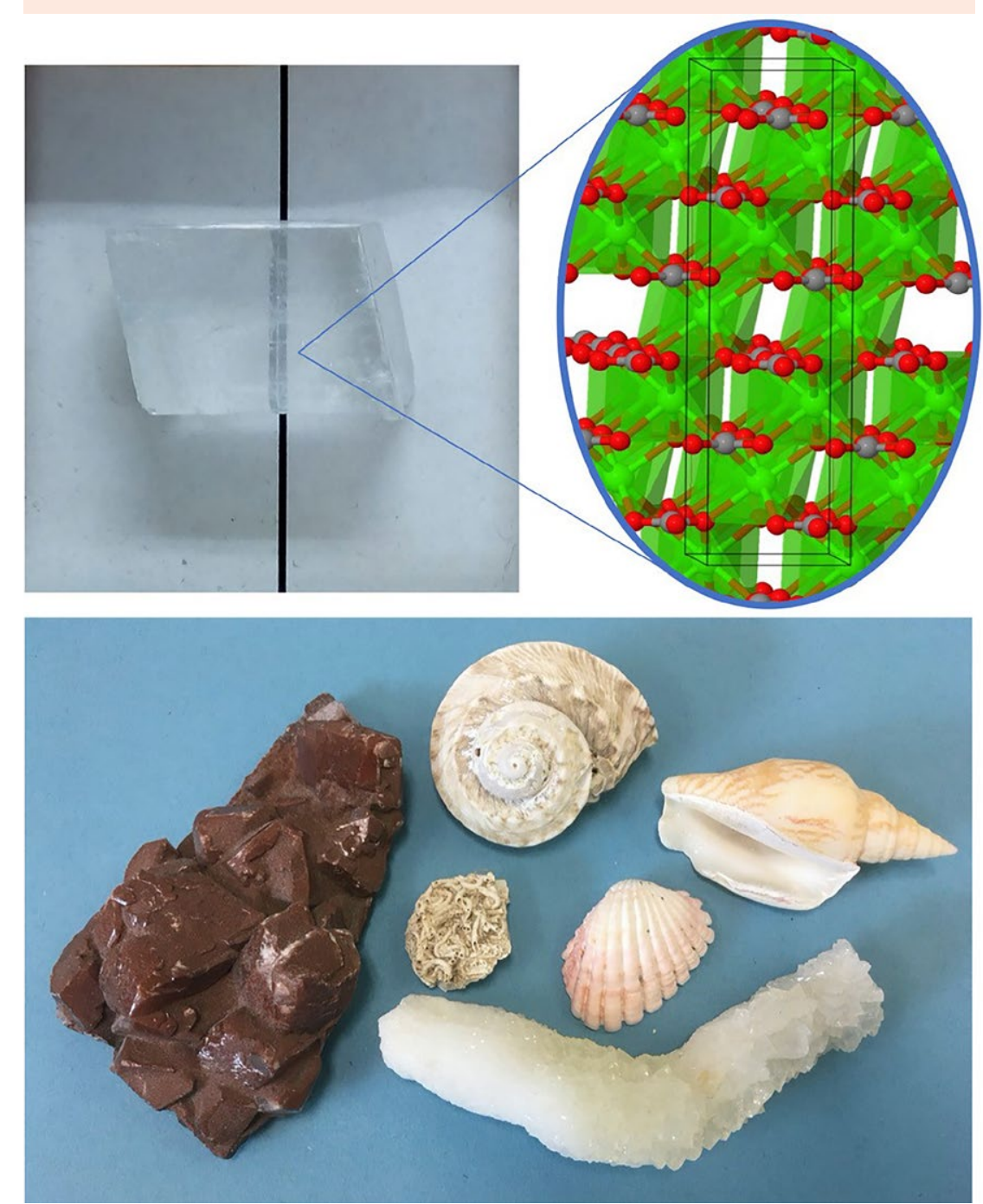

Fig. 1 Calcium carbonate minerals and biominerals. Top: a perfect crystal of calcite sitting on a
black line to emphasise its birefringence, with a representation of its atomic structure. Bottom:

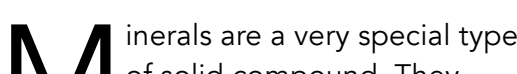
of solid compound. They chemical elements that are arranged Th actighly ordered fashion according to what is known as a crystal lattice. ogether with the composition, the then determine the properties and appearance of the mineral.

Common minerals include quartz, calcite, and haematite. The huge diversity in their appearance and properties - such as their hardness comes from their different chemical compositions and structure. Amethysts, specific type of quartz. Quartz is made get their rich colours from the presence incorporated with silicon and oxygen in the crystal.

There are numerous ways of forming new minerals. They can grow from minerals. Minerals can also be formed from magma as it cools or transformed that involve changes in temperature and pressure.

A wide variety of minerals are produced biologically, through a process that is called biomineralisation. Bone mineral, an inorganic crystalline solid known as hydroxyapatite, is what endows our skeletons with their strength and weight-bearing abilities. A number of animal pathologies are linked to bodies, such as kidney stones and wh their beauliful purple hues, are a of silicon and oxygen, but amethysts of irradiated iron impurities that are solutions, for example when waters leaving behind the residual dissolved into other minerals through processes steoporosis. Several sea creatures also produce minerals. Lobsters and other f their agregation of calcium and carbonate ons, resulting into calcium carbonate minerals (the same minerals that form scales in domestic devices and industria pipes). Many molluscs produce their shells and hard tissues in similar ways, and species like limpets make use of iron-containing minerals to form their terrifyingly strong teeth. While they may look inconspicuous, their teeth are made of one of the strongest known biologica material, and they make good use of them to cling on to rocks and scrape algae for feeding. Bacterias and plants are also able to grow minerals. For nample,

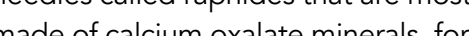
defence against herbivores.

The chemical and structural diversity of minerals makes them complicated to understand. For example, minerals with the same chemical composition can have different crystal structures and this impacts upon their properties, chemical reactivity, and appearance. Trying to untangle this complex science is at the heart of Dr Raffaella Demichelis' research at Curtin University, Australia. By using computational modelling, she is able to pinpoint with atomic precision what happens as minerals grow and react. From his, she is developing our understanding of why some minerals are so abundant to

\section{ATOMISTIC SIMULATIONS}

A very simple way of picturing how a collection of balls connected to the nearest neighbours by springs. Each. ball represents a chemical element. The springs between the balls represent the chemical bonding between the elements. Very strong chemical bonds are like strong, stiff springs that require ot of energy to move.

Although we cannot feel this movemen when we touch solid objects, all the moms in a mineral are in constan motion. The chemical bonds, or springs, oscillate at a particular frequency. One



Fon

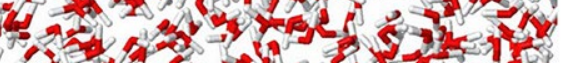

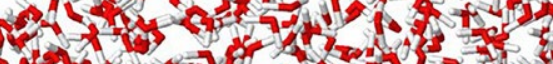

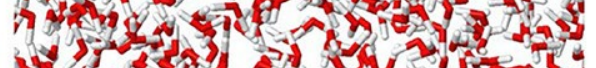

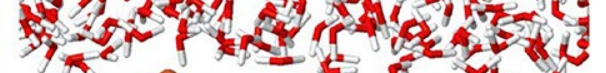

政

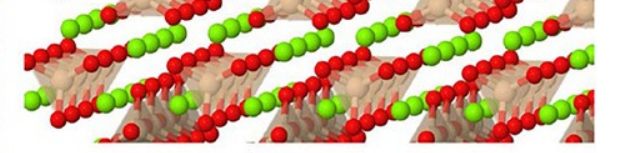

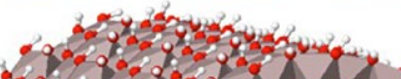

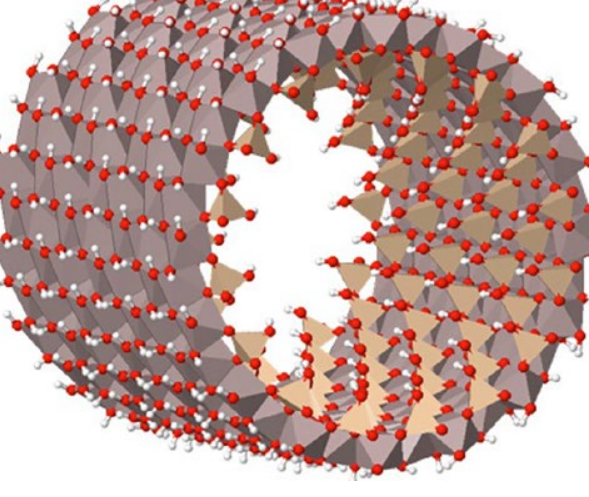

Fig. 2 Representation of the atomic structure of: vaterite interacting with a biomolecule - aspartic
acid - and water (top left); an iron-magnesium silicate interacting with liquid water (top right); a phosphate ion surrounded dy water (phosphate ions, together with calcium, make up the min
in our bones

With their simulations, Dr Demichelis" team could deduce the presence of magnesium impurities and determine the exact orientation of the water molecules.

atomistic simulations for solid materials is that a single mineral will be made of thousands, millions and even more a simulation, the more time it takes to compute or run the simulation.

Fortunately, researchers like Dr Demichelis have some tricks up their sleeves when dealing with such large systems and calculations. Although her calculations still require the use of very powerful supercomputing facilities, the regular and ordered nature of minerals mean that she can break down their structures into smaller, repeating patterns or motifs that make up the whole structure. From this, she can capture the level of detail and the individuat comes from describing mineral while making the calculations computationally feasible.

\section{MYSTERIES OF CALCIUM}

CARBONATES

One problem that has particularly captured the attention of Dr Demichelis and her research group are the mysteries around the structures of calcium carbonate minerals that are (c) Structures. The formh' and 'hydromorph' chetres. The former occur when the the same, but there are differences in the arrangement of atoms in the crysta structure; the latter occur when the chemical composition of the mineral is the same, but there are differences in the into the structure molecules incorpo 



Fig. 3 How and how strongly does calcium bind to aspartic acid - a biomolecule that makes up many proteins? Free energy plots of the association between calcium and two oforms of aspartic acid $(D$ and $L$ Lin water solu
separated by a layer of water molecules rather than in direct contact.

\section{assume 'amorphous' structures,}

according to a periodic lattice onised

The most stable and therefore most abundant forms of calcium carbonate are calcite and aragonite. However, hydromorphs and amorphous phases exist. Recently, one such hydromorph

structure, which would look different in case of a pure calcium phase.

\section{A RARE FORM}

Not all mineral forms are equally studying one of the least common types , a mineral known living organisms, including plants, fish

biologically active molecules can direct the formation of calcium carbonate, something particularly relevant for vaterite as it is found most frequently in biological environments.

For example, her team used these types of simulations to look at which surfaces of the mineral are stable in water. This is because most biological discovered during There are several different forms of to mineralisation an experiment aimed at studying calcium carbonate, each having different From this, she $\begin{array}{lll}\begin{array}{l}\text { the formation of } \\ \text { amorphous calcium }\end{array} \quad \text { crystalline or amorphous structure. } & \begin{array}{l}\text { could also track } \\ \text { exactly how water }\end{array}\end{array}$ carbonate. This

(a)

By using atomistic simulation, determine the exact wasitions to orientations of the water molecules calcium carbonate hemihydrate while also demonstrating that the structure experimentally observed contain magnesium impurities. Such phase was indeed originally thought as a pure calcium phase.

By simulating several possible structures of calcium carbonate hemihydrate, with and without the presence of
magnesium, Dr Demichelis was able to conclude th Demichelis was able to conclude that magnesium impurit
cause a distortion of the crystal freshwater snails, and saltwater clams. It is the mineral many molluscs produce epair their shells when damaged. Unlike many other calcium carbon vaterite has been debated for decads without reaching an agreement. Dr Demichelis' calculations shed a light on this complex mineral by proposing a solution for its structure that has been confirmed also experimentally.

In her simulations, Dr Demichelis can explore not just how the arrangement of atoms in space affects a mineral's properties and behaviour, but also simulate how these evolve over time. the challenge of modelling how with vaterite, how long each molecule spends attached to the surface before in, and detect and analyse possible attachment sites for biomolecules.

Determining how biological molecules can direct the formation of minerals is crucial for understanding how lifeforms like limpets can produce some of the world's most impressive materials. Living organisms are able to grow minerals with specific sizes, compositions and shapes, something far beyond our limits be able to mimic this incredible prowess at materials chemistry, first we need to understand in detail how it works, research helps us to do. Demichelis' in materials synthesis. If we are going to

\section{(28) Eehind the Research}

Dr Raffaella Demichelis

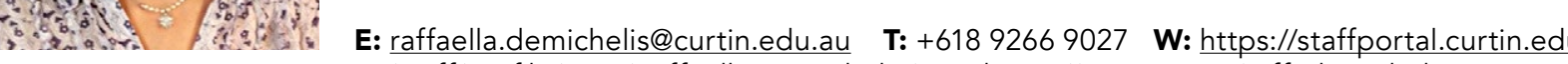
au/staff/profile/view/Raffaella. Demichelis/ $\square$ https://twitter.com/raffademichelis

\section{Research Objectives}

Dr Raffaella Demichelis uses atomistic simulations to (ther environment, and to explain their properties.

\section{Detail}

\section{Raffaella Demichelis}

Bio

Dr Raffaella Demichelis is an award-winning scientist who leads a team at Curtin University doing research in materials chemistry and geochemistry, using computational techniques. She engages with STEM outreach activities and advocates for a mentally safe, flexible and inclusive research environment, allowing for sustainable and diverse career paths.

\section{Funding}

Cosearch Council

- Curtin University

\section{Collaborators}

Pawsey Centre (facility)

nal Infrastructure (facility)

- Dr Natalya Garcia

- Dr Katarzyna Koziara

- Dr Alicia Schuitemake

- DrWen Zhao

- Prof Paolo Raiteri

- Prof Julian Gale

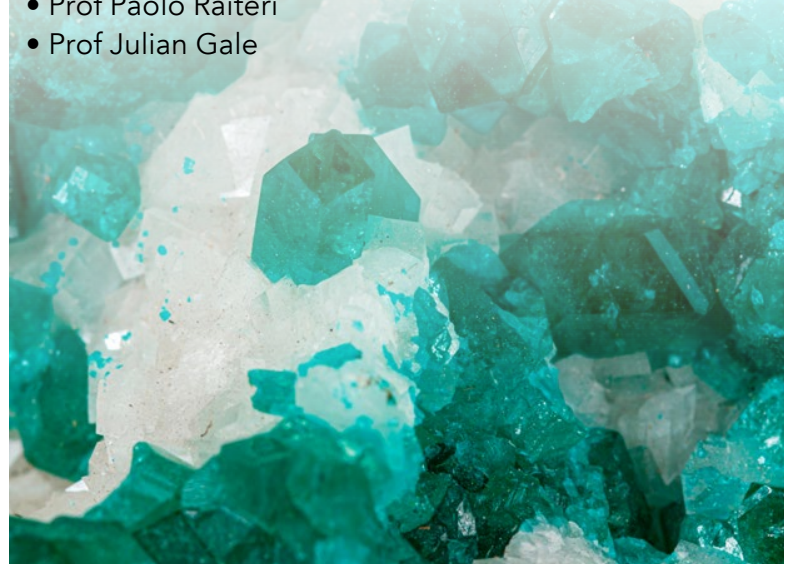

\section{References}

- Schuitemaker, A., Raiteri, P., \& Demichelis, R. (2021). The atomic structure and dynamics at the $\mathrm{CaCO} 3$ vateritewater interface. A classical molecular dynamics study. The Journal of Chemical Physics, 154(16), 164504. Available at: https://doi.org/10.1063/5.0049483 - Aufort, J., \& Demichelis, R. (2020). Magnesium Impurities Crystal Growth and Design, 20(12), 8028-8038. Available at: https://doi.org/10.1021/acs.cgd.0c01282

- Demichelis, R., Garcia, N. A., Raiteri, P., Innocenti Malini, R., Freeman, C. L., Harding, J. H., \& Gale, J. D. (2018). Simulation of Calcium Phosphate Species in Aqueous Solution: Force Field Derivation. Journal of Physical Chemistry B, 122(4), 1471-1483. Available at: https://doi. org/10.1021/acs.jpcb.7b10697

- Demichelis, R., Raiteri, P., Gale, J. D., \& Dovesi, R. (2013). The Multiple Structures of Vaterite. Crystal Growth and Design, 13(6), 2247-2251. Available at: https://doi. org/10.1021/cg4002972 - Demichelis, R., Raiteri, P., Gale, J. D., Quigley, D., \& Gebauer, D. (2011). Stable prenucleation mineral clusters are liquid-like ionic polymers. Nature Communications, 2(1), 590. Available at: https://doi.org/10.1038/ ncomms 1604

\section{Personal Response}

\section{What types of minerals will you be looking at in}

II My group is starting to look into the properties their potential applicability to harvest carbon dioxide from our atmosphere and eventually transform it into molecules of economic or biological significance - all this while continuing to unravel the mysteries behind the formation of biominerals like carbonates, phosphates

\section{Curtin University}

